\title{
Mejora de la etapa de llenado en moldes de inyección de plástico usando vibración
}

\section{Improvement Performance of the Filling Step in Injection Mold through Vibration}

\author{
Benítez-Rangel J.P. \\ Facultad de Ingeniería \\ Universidad Autónoma de Querétaro, San Juan del Río \\ Correo:benitez@uaq.mx \\ Morales-Hernández L.A. \\ Facultad de Ingeniería \\ Universidad Autónoma de Querétaro, San Juan del Río \\ Correo:luis_morah@yahoo.com
}

\author{
Trejo-Hernández M. \\ Facultad de Ingeniería \\ Universidad Autónoma de Querétaro, San Juan del Río \\ Correo:mtrejo@hspdigital.org
}

Información del artículo: recibido: marzo de 2009, aceptado: octubre de 2011

\section{Resumen}

El presente trabajo muestra la mejora de flujo que tiene la etapa de llenado del proceso de inyección de plásticos, debida a la excitación del polímero a través de vibración. Dicho proceso, puede resumirse en tres etapas principales que son: el llenado, el empaquetado y el enfriamiento. El proceso de llenado es el paso en el que se pueden cambiar una gran cantidad de propiedades, tanto mecánicas como estéticas del producto terminado. El objetivo de esta investigación es mostrar que el llenado del molde mejora adicionando vibración, sin tener que agregar aditivos químicos. Para ello, se llevó a cabo el diseño y fabricación de un molde experimental, en el cual se acondicionó un mecanismo de vibración que permitió demostrar las ventajas de la vibración en dicho proceso. Además, se propuso una metodología heurística para la elaboración de las pruebas que reveló una mejora en el llenado con frecuencias cercanas a los $3 \mathrm{~Hz}$.

\footnotetext{
Abstract

This paper shows the flow improvement in the filling step of the polymer injection process due to the polymer excitation though vibration. This process can be split up into three main steps: filling, pocking and cooling. Several mechanical and aesthetic properties of the finished product can be changed in the filling step. The objective of this investigation is to demonstrate the improvement in the filling mold under vibration without adding chemical products. To reach this result, an experimental mold was designed and manufactured in which a vibration device was coupled; it was possible to demonstrate the vibration advantage through this process. Moreover, a heuristic methodology was proposed for the experiment which shows an improvement in the filling process with frequencies close to $3 \mathrm{~Hz}$.
}

\section{Descriptores:}

- inyección de plástico

- reología, vibraciones

- manufactura de moldes

- llenado de molde

- polímeros

\section{Keywords:}

- vibration mold,

- injection process, rehology,

- polymers

- plastic mold

- mold filling 


\section{Introducción}

Actualmente existen un sin fin de productos químicos que permiten mejorar el proceso de moldeo por inyección; sin embargo, muchos de ellos son productos consumibles, lo que implica un gasto constante, por otro lado, estos aditivos funcionales y de proceso como depresores de viscosidad, deslizantes lubricantes, plastificantes, entre otros, modifican el peso molecular y con ello cambian las propiedades mecánicas propias del polímero. Por tal motivo es deseable contar con un método no-químico que modifique la viscosidad del polímero, por lo menos en el tiempo en que tarda en ser moldeado, sin tener que sacrificar las propiedades del polímero.

Con el objeto de mostrar las mejoras del proceso, es necesario desarrollar un aparato para modificar la viscosidad a través de vibración. Es importante aclarar, que este desarrollo es novedoso con respecto a otros aparatos existentes como el desarrollado por J.P. Ibar, bajo la patente americana 5885495 en 1999, o el de Liu en 2006, entre otros, que explotan el mismo principio de vibración. Sin embargo, la relevancia tecnológica radica en que el desarrollo que aquí se muestra es un accesorio del molde y no el desarrollo o modificación del sistema de inyección de una máquina.

Haciendo un análisis de los resultados alcanzados con el uso de vibración en los procesos de manufactura, como extrusión y fundición, es posible inferir que su aplicación en la inyección de plásticos puede mostrar buenos resultados. Más aún, los avances tecnológicos permiten la modelación previa de los procesos con una insignificante pérdida de tiempo.

Por ejemplo, Liu (2006) analiza el comportamiento del polietileno en un proceso de extrusión adicionando vibración senoidal. Por otro lado, Xiaochun y Jinping (2006) encuentran mejoras relacionadas con la vibración asistida en el proceso de inyección de polvos de metal (MIM, metal powder injection). En el campo de la fundición, Abu et al. (2005) muestran que la vibración del molde en el momento de la colada mejora la morfología de la aleación AlSi. El proceso de inyección consta de tres pasos principales: llenado, empaquetamiento y enfriamiento (Benítez, 2007). El llenado es el paso en que se puede tener una mayor influencia sobre las propiedades de las piezas moldeadas, como estabilidad dimensional, brillo superficial y resistencia mecánica, entre otras. Wang et al. (2006) estudiaron la relación entre el efecto de la vibración en el proceso de inyección de polipropileno con sus propiedades mecánicas, por mencionar algunas de las investigaciones más recientes.
Aunque en los últimos años se ha invertido un considerable esfuerzo para desarrollar sistemas que integren vibración, gran parte de ellos son poco prácticos, por ejemplo, tanto las investigaciones de Xiaochun y Jinping (2006) como las de Liu (2005) tienen la desventaja de que el aditamento desarrollado requiere montarlo sobre la inyectora, no sobre el molde, lo cual no es fácil de implementar en la industria, es decir, los costos industriales de modificar una inyectora son mucho mayores que los de modificar un molde. Por lo que el aparato desarrollado como parte de la metodología facilita la aplicación industrial.

En resumen, en este trabajo se propone un método innovador para mejorar el ciclo de llenado del proceso de inyección, exponiendo al polímero fundido mientras se llena la cavidad a oscilaciones senoidales de determinada frecuencia y a una temperatura constante, con el fin de mejorar la etapa del llenado del molde.

Un vástago colocado en el bebedero del molde excita al polímero fundido y un vibrador (shaker) ubicado fuera del molde y la máquina de inyección excita al vástago.

La innovación del sistema vibrador sobre las otras técnicas consiste en que no es estrictamente necesario modificar la inyectora, en otras palabras, es un accesorio que se instala en el molde (figura 4), lo que permitirá una más rápida integración de esta tecnología al campo de trabajo.

\section{Estado del arte}

Existen muchas variables que se pueden cambiar para modificar la viscosidad de un polímero fundido, entre las cuales destacan:

Efecto de la temperatura en la viscosidad del plástico fundido

Un factor que puede ayudar para la disminución de la viscosidad en los fluidos es el aumento de temperatura, ya que provoca un movimiento (mayor separación) de las cadenas poliméricas, disminuyendo la intensidad de las fuerzas de cohesión, generando así la disminución de la viscosidad.

La manera de comprobarlo es observar algunas de las fórmulas que permiten ver la relación existente entre la viscosidad de un líquido y su temperatura. Las más importantes son:

a) Ecuación de Arrhenius: la ecuación (1) es una expresión matemática que nos ayuda a comprobar la dependencia de la constante de la viscosidad dinámica 
con la temperatura y las constantes dependientes del líquido.

$\mu=A^{*} e^{\frac{B}{T}}$

donde

$\mu$ es la viscosidad dinámica,

$A$ y $B$ son constantes dependientes del líquido y

$T$ es la temperatura absoluta del polímero.

b) La ecuación de Poiseuille:

$\mu=\frac{\mu_{0}}{1+\alpha T+\beta T^{2}}$

donde

$\mu_{0}$ es la viscosidad dinámica a $0^{\circ} \mathrm{C}$,

$T$ la temperatura del polímero y

$\alpha$ y $\beta$ coeficientes constantes.

Como se ve en las ecuaciones (1) y (2), la viscosidad disminuye con el aumento de temperatura. Esto se debe al hecho de que, al aumentar la temperatura, la energía cinética supera a las fuerzas viscosas, dando lugar a una disminución de la viscosidad.

Por este hecho se deben extremar las precauciones a la hora de medir la viscosidad, teniendo en cuenta que la temperatura del polímero debe permanecer prácticamente constante.

Efecto de la velocidad de flujo en la viscosidad del plástico fundido

La viscosidad no constante, que depende de la velocidad de cizalla, es precisamente lo que da a los polímeros el carácter no-Newtoniano, si la viscosidad permaneciera constante a pesar del cambio de velocidad, el comportamiento seria Newtoniano.

$\mu=\tau / D$

despejando $\tau$ de la ecuación (3), se tiene:

$\tau=\mu^{*} D$

donde

$\tau$ es el esfuerzo cortante,

$D$ la velocidad de corte $y$

$\mu$ la viscosidad.

Se observa en la ecuación (4) que la velocidad está directamente relacionada con la viscosidad, es decir, a mayor velocidad de flujo (por lo tanto, velocidad de corte) menor viscosidad.

A velocidades de flujo muy bajas (figura 1a) la mayoría de las moléculas están enmarañadas o, dicho de otra forma, existe un alto grado de fricción entre ellas. A medida que la velocidad de flujo aumenta (figura $1 b$ ), las moléculas empiezan a desenmarañarse y a orientarse. Las moléculas orientadas están menos enmarañadas, de manera que la fricción entre ellas se reduce y ahora fluyen con más facilidad. Debido a que existe menos fricción entre ellas, la viscosidad del plástico disminuye, es decir, la viscosidad del plástico disminuye al aumentar la velocidad de flujo (Yañez y Sánchez, 2001).

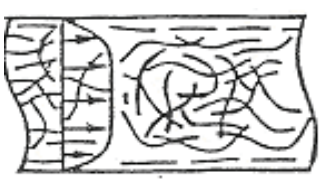

a)

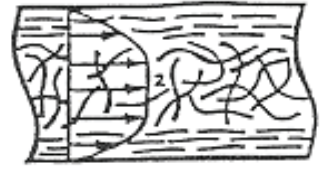

b)
Figura 1. Efecto de la velocidad en el ordenamiento de las partículas

Efecto de la vibración en la viscosidad del plástico fundido

La reología juega un papel importante en el tratamiento de los polímeros. El comportamiento de un polímero fundido puede cambiar enormemente por la aplicación externa de fuerzas durante su inyección

Se acepta ampliamente, y se puede percibir en los casos anteriores, que la disminución de la viscosidad favorece la correcta orientación o desenmarañamiento de las cadenas poliméricas (Zheng et al., 2002)

La aplicación de vibración reduce las fuerzas de unión de las partículas y facilita la alineación de las cadenas poliméricas, con lo que se puede controlar el comportamiento del polímero y obtener productos con una mayor calidad. Cuando la dirección de flujo del polímero fundido es paralela con la vibración, el efecto es mucho más fuerte en comparación con la posición ortogonal; sin embargo no es limitante y facilita el diseño del mecanismo.

En 2005, Liu investigó la relación entre la viscosidad y la variación de frecuencia (ecuación 5), pero sólo se probó para extrusión.

$\eta_{a}=\frac{\pi R^{3}}{\bar{Q}} \cdot \frac{a \cdot \Delta p}{3+\frac{a^{2} \cdot \Delta p^{2} \cdot \varepsilon p \cdot \varepsilon q \cdot \cos \varphi}{a^{2} \cdot \Delta p^{2} \cdot \varepsilon_{p}{ }^{2}+2 a \cdot b \cdot \Delta p \cdot \bar{Q} \cdot \varepsilon p \cdot \varepsilon p \cdot \omega \cdot \sin \varphi+b^{2} \cdot \bar{Q}^{2} \varepsilon_{q}{ }^{2} \cdot \omega^{2}}}$ 
donde las principales variables son:

$\eta_{a}$ viscosidad aparente,

$P$ presión,

$Q$ tasa de capilaridad y

$\omega$ frecuencia.

Bajo la influencia de vibración, el número total de puntos de unión entre las cadenas poliméricas disminuye, entonces, la viscosidad del polímero baja. Sin embargo, debe tenerse cuidado en la selección de la frecuencia, ya que si la vibración es excesiva, puede favorecer a un enredamiento de partículas que resulta en un leve incremento de viscosidad, contrario a lo que se desea y se pierde el efecto esperado (Zheng et al., 2002).

\section{Metodología}

En esta sección se muestra tanto el diseño del experimento como la manufactura de equipos auxiliares para su desarrollo como el molde y el excitador.

\section{Diseño de molde}

En este trabajo es necesario mostrar el cambio de viscosidad, por lo que el molde en sí constituye un viscosímetro no convencional, según la propuesta de Clavería et al. (2005), a través del cual se demostrará experimentalmente dicho cambio.

Por tal motivo, el diseño que se usó es un molde con forma de espiral. Dicho molde puede responder a diferentes matrices o patrones, en este caso se seleccionó una espiral de Arquímedes (ecuación 6); lo anterior se debió a que ésta tiene una gran cantidad de características como la distancia entre espiras que es constante, la existencia de un método, tanto analítico como práctico para medir su longitud y, de la misma manera, la forma de su trazo responde a una ecuación matemática o a una representación gráfica simple.

En coordenadas polares $(r, \theta)$ la espiral de Arquímedes puede describirse por la ecuación (6):

$R=\theta^{*} a$

donde

$\mathrm{R}$ es el modulo o distancia al origen,

a una constante real y

$\theta$ el desplazamiento angular.

La figura 2 muestra el molde que se utilizó en el experimento, dicho molde fue maquinado en acero 1018 (cold rolled), con dimensiones de $254 \mathrm{~mm} \times 457 \mathrm{~mm}$. Para obtener una mayor precisión, la espiral de Arquímedes fue programada y maquinada en un centro de mecanizado CNC.

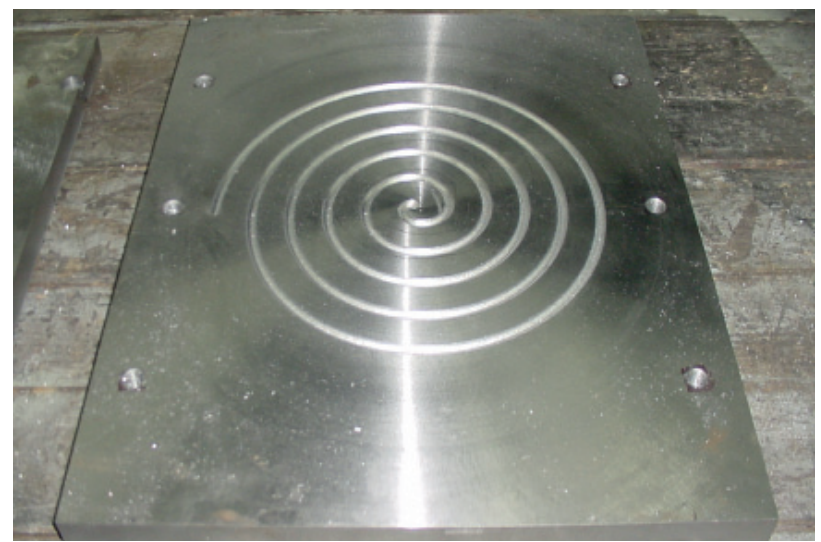

Figura 2. Molde de inyección en espiral

\section{Diseño del excitador}

Otra de las herramientas desarrolladas para llevar a cabo las pruebas es el mecanismo de vibración, el cual excitará el polímero fundido. Dicho mecanismo de excitación se coloca en la boquilla de inyección por varias razones, una de ellas es favorecer que el contacto con el fluido sea lo más directo posible, por otro lado, a pesar de que el vibrador está colocado de manera perpendicular al flujo, esta colocación favorece la sencillez del instrumento (figura 3), ya que de lo contrario se tendría que modificar el cañón de inyección para su montaje como lo sugiere Liu $(2005,2006)$.

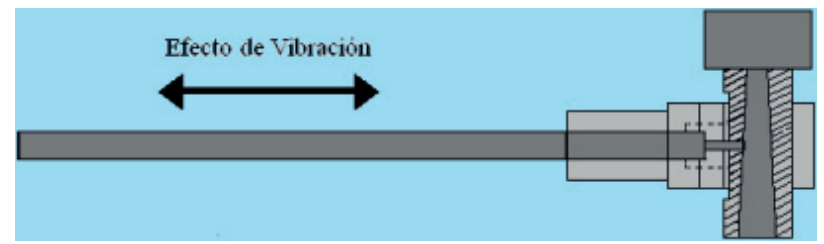

Figura 3. Mecanismo transductor

La oscilación del mecanismo se genera a través de una leva ajustable que controla la amplitud y la frecuencia. La leva se excita con un motor CA, que se controla a través de un variador de frecuencia (figura 4).

\section{Preparación del experimento}

Para este experimento se utiliza polietileno (HDPE PEAD 60120 de PEMEX) ya que es un polímero con buenas propiedades físicas y que no tiene mayores problemas en la inyección, como de absorción de humedad, entre otras. Algunas de las propiedades que 


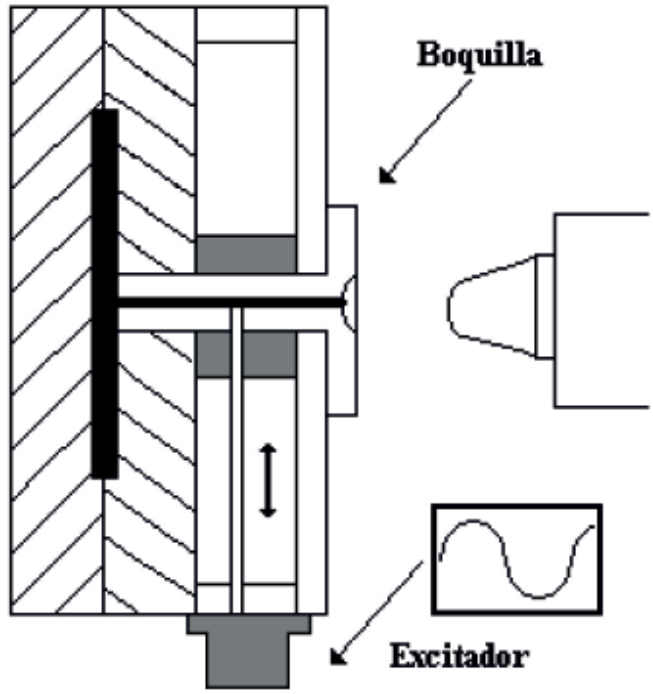

Figura 4. Componentes del excitador

hacen del polietileno una materia prima muy conveniente para miles de artículos manufacturados son: poco peso, flexibilidad, tenacidad, alta resistencia química y propiedades eléctricas sobresalientes (Guerrero et al., 2003).

El polímero se inyecta usando una máquina marca Husky de 200 Ton. La temperatura ajustada en la máquina para el experimento fue de $140^{\circ} \mathrm{C}$. El ciclo de inyección fue semiautomático con un tiempo de duración de $7 \mathrm{~s}$. Debido a la cantidad de ciclos del experimento y al tamaño de pieza, no fue necesario utilizar un sistema de enfriamiento para el molde.

La metodología que se siguió en el experimento fue la siguiente: las variables de temperatura, velocidad y tiempo permanecen constantes, lo único que cambia de una prueba a otra es la aplicación de la vibración en el proceso de llenado. Se lleva a cabo un ciclo de 6 pruebas para cada frecuencia, éstas son 2, 3, 4, 5, 6, 7, $919 \mathrm{~Hz}$, elegidas de manera heurística, con una amplitud de $0.15 \mathrm{~mm}$.

En cuanto a los resultados, se medirán sólo a través de comparación, tanto en longitud como en peso, entre una prueba que no tuvo vibración y otra donde sí la hubo.

Lo que se observa en el experimento es el desplazamiento del fluido dentro del molde. La forma de obtener los resultados es medir el desplazamiento con un transportador calibrado en grados.

En cada prueba se determina el peso del polímero inyectado con el afán de comprobar que en una muestra en que se usó vibración, la cantidad de plástico inyectado es mayor en comparación con la de una muestra donde no se usó.

\section{Resultados y discusión}

A continuación se muestran los desarrollos hechos para el proyecto como el molde en espiral, mecanismo de acoplamiento y mecanismo excitador, como se muestra en las figuras 5,6 y 7 , respectivamente.

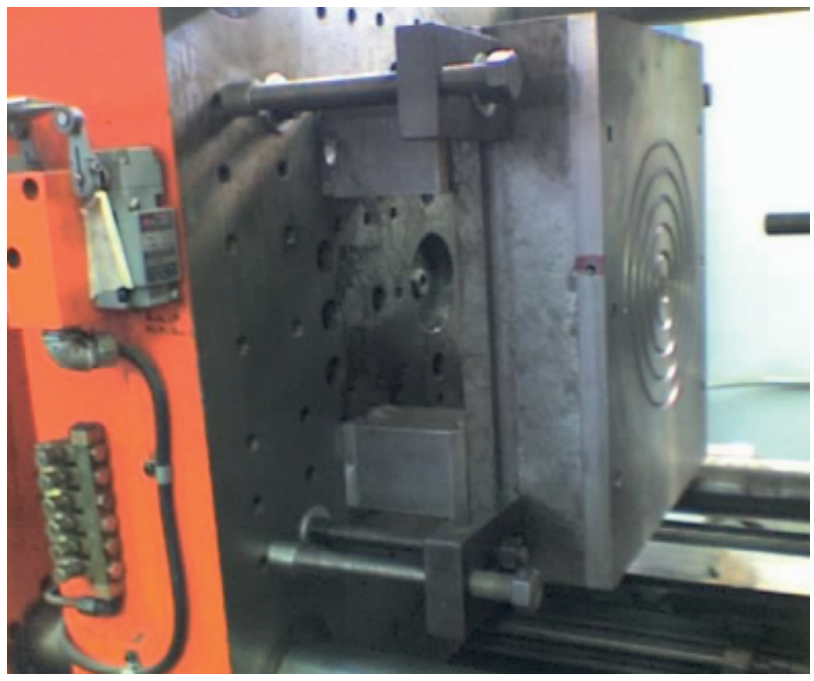

Figura 5. Molde en espiral usado en las pruebas

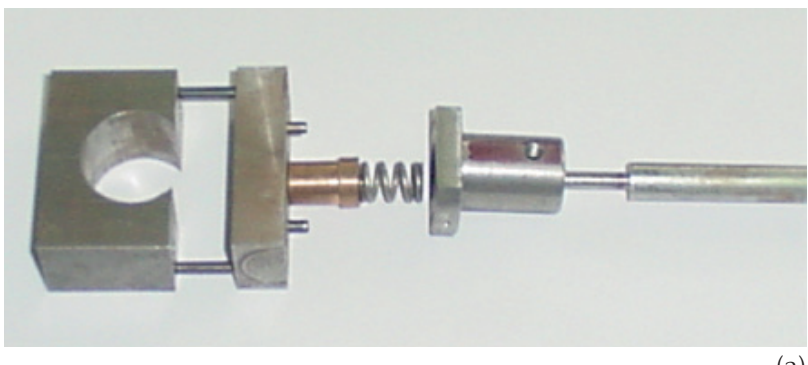

(a)

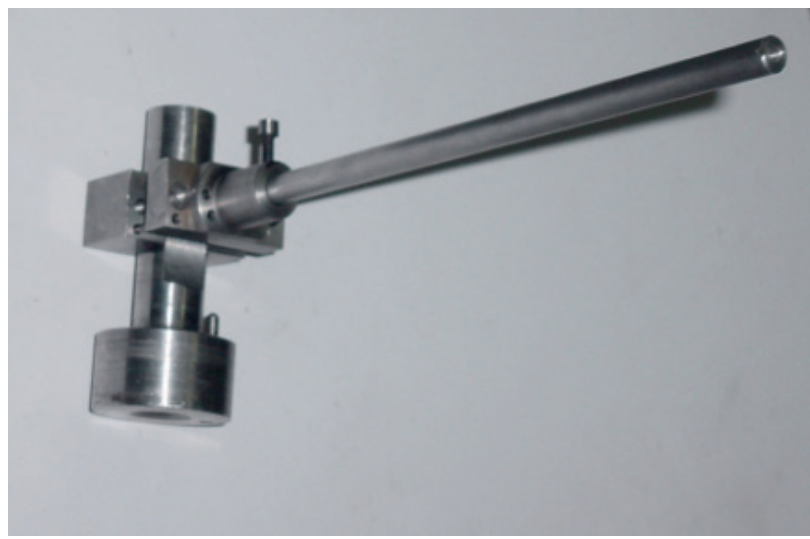

(b)

Figura 6. Mecanismo acoplador entre la boquilla de inyección y el excitador 


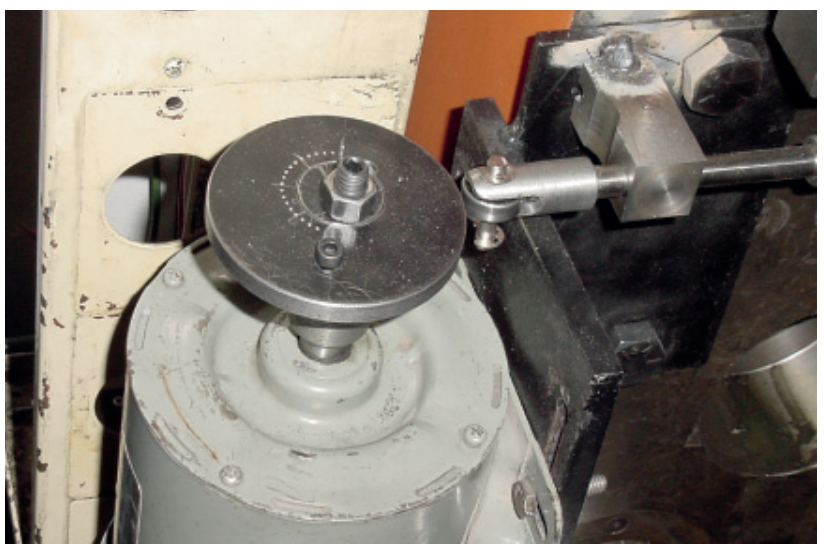

Figura 7. Mecanismo excitador de leva ajustable

Se llevó a cabo el experimento (figura 8) en el que se pudo constatar el efecto positivo de la vibración al momento de la inyección de plástico. La aplicación desarrollada no hizo necesario modificar toda la máquina, ya que sólo bastó con agregar un mecanismo sencillo de vibración en la boquilla de inyección del molde para obtener la mejora en el llenado del molde. El proceso fue un método no-químico que modificó la viscosidad del polímero, por lo menos durante el tiempo que tomó moldearlo, sin tener que sacrificar las propiedades del polímero. Finalmente se muestra que la frecuencia óptima para trabajar con el excitador fue de $3 \mathrm{~Hz}$.

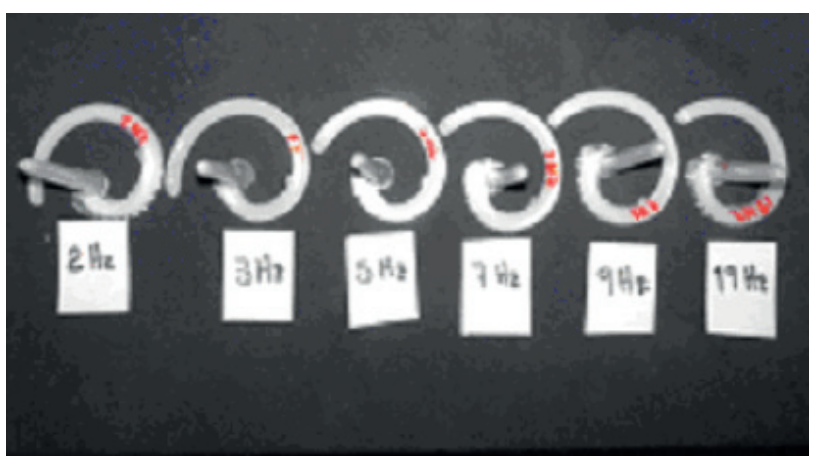

Figura 8. Espirales inyectadas a diferentes frecuencias
La tabla 1 muestra algunos de los resultados de desplazamientos angulares a diferentes frecuencias, así como la diferencia entre la inyección con vibración y sin ella.

La figura 9 muestra en su eje horizontal las frecuencias a las que se realizaron los experimentos y en su eje vertical la diferencia, medida en desplazamiento angular, de una inyección con vibración y sin ella. Se muestra el evidente incremento de desplazamiento en frecuencias bajas, donde el mayor se obtuvo en $3 \mathrm{~Hz}$. Es posible observar también que a frecuencias altas no se observa un incremento notable, lo mismo que a frecuencias cercanas a cero.

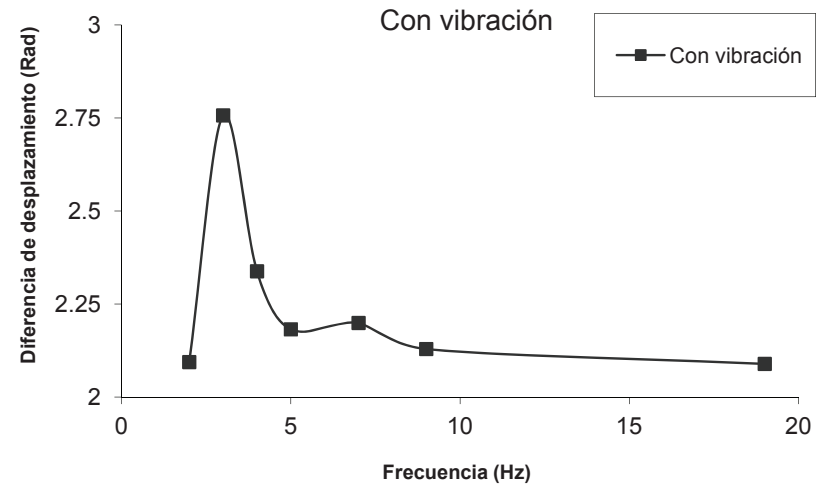

Figura 9. Diferencial de desplazamiento de las espiras

Otra manera en que se puede observar el cambio en las muestras es midiendo la masa inyectada. Las muestras se pesaron y los resultados se observan en la tabla 2. Para obtener una mejor lectura las medidas se realizaron en onzas $[\mathrm{Oz}]$. En esta misma tabla se presenta la incertidumbre estándar para cada grupo de experimentos. La figura 10 muestra el resultado gráfico de este experimento.

\begin{tabular}{cccc}
\hline $\begin{array}{c}\text { Desplazamiento angular } \\
\text { sin vibración (Rad) }\end{array}$ & $\begin{array}{c}\text { Desplazamiento angular } \\
\text { con vibración (Rad) }\end{array}$ & Frecuencia Hz & $\begin{array}{c}\text { Diferencia } \\
(\mathrm{Rad})\end{array}$ \\
\hline 2.076 & 2.094 & 2 & 0.018 \\
2.076 & 2.757 & 3 & 0.681 \\
2.076 & 2.338 & 4 & 0.262 \\
2.076 & 2.182 & 5 & 0.106 \\
2.076 & 2.199 & 7 & 0.123 \\
2.076 & 2.129 & 9 & 0.053 \\
2.076 & 2.089 & 19 & 0.013 \\
\hline
\end{tabular}

Tabla 1. Diferencial de desplazamientos 


\begin{tabular}{ccccc}
\hline $\begin{array}{c}\text { Peso sin vibración } \\
(\mathrm{Oz})\end{array}$ & $\begin{array}{c}\text { Peso con vibración } \\
(\mathrm{Oz})\end{array}$ & $\begin{array}{c}\text { Incertidumbre } \\
\%\end{array}$ & $\begin{array}{c}\text { Frecuencia } \\
\mathrm{Hz}\end{array}$ & $\begin{array}{c}\text { Diferencia } \\
(\mathrm{Oz})\end{array}$ \\
\hline 0.18 & 0.18 & 0.100 & 2 & 0 \\
0.18 & 0.1898 & 0.088 & 3 & 0.0098 \\
0.18 & 0.1842 & 0.033 & 4 & 0.0042 \\
0.18 & 0.1842 & 0.220 & 5 & 0.0042 \\
0.18 & 0.1828 & 0.142 & 7 & 0.0028 \\
0.18 & 0.1815 & 0.044 & 9 & 0.0015 \\
0.18 & 0.1803 & 0.033 & 19 & 0.0003 \\
\hline
\end{tabular}

Tabla 2. Diferencial másico

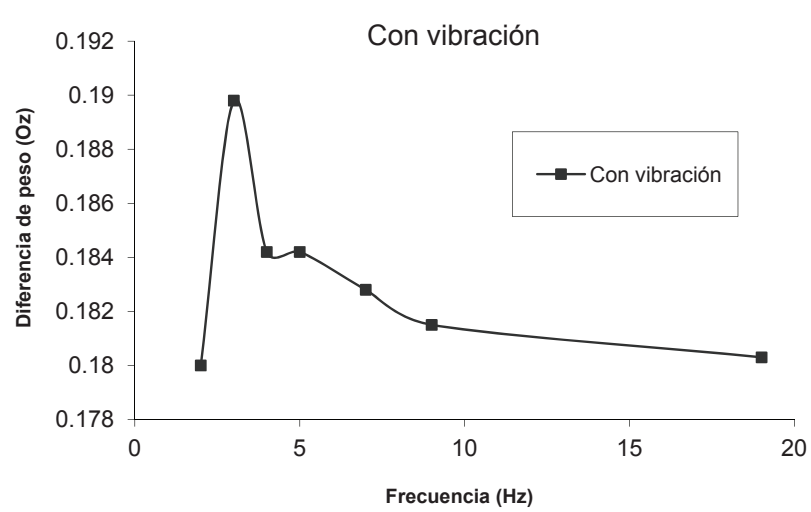

Figura 10. Gráfica de diferencial másico

Es obvio que al entrar una cantidad mayor de plástico en la cavidad, la pieza debe pesar más, por lo que la gráfica de la figura 10 confirma que a la frecuencia de 3 $\mathrm{Hz}$ se logra inyectar una mayor cantidad de polímero y por consiguiente existe una relación de comportamiento entre el resultado mostrado en las figuras 9 y 10 a la frecuencia mencionada.

\section{Conclusiones}

En este trabajo se desarrolló un método novedoso y de fácil aplicación industrial, no químico, para modificar la viscosidad de un polímero dentro del proceso de inyección de plásticos. El método se basó en la adición de vibración al polímero durante el proceso de inyección.

En la primera parte de la investigación se hizo un análisis de las ecuaciones que toman parte en el proceso de mejora de la inyección plásticos, poniendo especial énfasis en la etapa de llenado del molde. Se encontró que la viscosidad puede cambiarse por temperatura, velocidad y vibración, donde ésta última es la que se demuestra en este trabajo.

La segunda parte contiene la demostración experimental de que excitando el polímero mediante vibración, se modifica la viscosidad. Es decir, se realizó la inyección de una pieza muestra, en este caso una espiral trazada bajo la ecuación de Arquímedes, que permitió medir el fenómeno viscosidad-vibración usando un método no convencional.

Los resultados obtenidos demuestran la contribución positiva de la vibración al proceso de inyección a frecuencias cercanas a $3 \mathrm{~Hz}$. Se reportó la dispersión obtenida para cada bloque de experimentos a cada frecuencia obteniendo la estimación de la incertidumbre estándar aceptable entre cada frecuencia (menor que 1\%). La aplicación demostró ser una buena opción como método no químico de modificación de viscosidad.

\section{Agradecimientos}

Los autores desean agradecer al CONACYT por el financiamiento de sus estudios de posgrado. Y al M.C. Abdiel García-Moreno por el apoyo brindado en el desarrollo de la etapa de pruebas.

\section{Referencias}

Abu-Dheir N., Khraisheh M., Saito K., Male A. Silicon Morphology Modification in the Eutectic Al-Si Alloy Using Mechanical Mold Vibration. Materials Science \& Engineering. A., volumen 393, 2005.

Benitez-Rangel J.P., Domínguez-González A., Herrera-Ruiz G., Delgado-Rosas M. Filling Process in Injection Mold: A Review. Polymer-Plastics Technology and Engineering, volumen 46, 2007.

Clavería I., Javierre C., Ponz L. Meted for Generation of Rehological Model to Caracterize Non-Conventional Injection Molding by Means of Spiral Mold. Journal of Material Processing Technology, volumen 162 (número 163), 2005.

Guerrero C., Lozano T., González V., Arroyo E. Morfología y propiedades del politereftalato de etilen-glicol y polietileno de alta densidad. Ciencia UANL, volumen 6, 2003.

Liu Y.J. Rehological Equation for Polymer Melt under the Action of Vibration. Plastic, Rubbers and Composites, volumen 34, 2005. 
Liu Y.J. Elastic Behavior Analysis of Polymer Melt Extruding Trough Capillarity with an Additional Sinusoidal Vibration. Polymer Bulletin, volumen 56, 2006.

Wang Q., Jin-Ping Q. y He L. Effect of Vibration Parameters of Electromagnetic Dynamic Plastics Injection Molding Machine on Mechanical Properties of Polypropylene Samples. Journal of Applied Polymer Science, volumen 102, 2006.

Xiaochun Y., Jinping Q. Experimental Study of the Vibration-Assisted MIM Process on Cavity Presure. Journal of Thermoplastic Composite Materials, volumen 19, 2006.

Yañes I., Sánchez S. Moldeo por inyección de plásticos. Centro de investigación en química aplicada, Saltillo, Coah., 2001.

Zheng Y., Kai Z.S., Jie Z., Li M.C., Chixing Z. Effect of Vibration on Rheology of Polymer Melt. Journal of Applied Polymer Science, volumen 85, 2002.

\section{Este artículo se cita:}

\section{Citación Chicago}

Benítez-Rangel, Juan Primo, Luis Alberto Morales-Hernández, Miguel Trejo-Hernández. Mejora de la etapa de llenado en moldes de inyección de plástico usando vibración. Ingeniería Investigación y Tecnología XIII, 04 (2012): 403-410.

\section{Citación ISO 690}

Benítez-Rangel J.P., Morales-Hernández L.A., Trejo-Hernández M. Mejora de la etapa de llenado en moldes de inyección de plástico usando vibración. Ingeniería Investigación y Tecnología, volumen XIII (número 4), octubre-diciembre 2012: 403-410.

\section{Semblanza de los autores}

Juan Primo Benítez-Rangel. Ingeniero electromecánico por la Universidad Autónoma de Querétaro (UAQ), maestro en instrumentación y control automático por la UAQ y doctor en ingeniería mecánica por la misma institución. Actualmente es profesor investigador de la Facultad de Ingeniería de la UAQ. Sus áreas de desarrollo son procesos de manufactura, moldes de inyección y automatización.

Luis Alberto Morales-Hernández. Profesor-Investigador de la Facultad de Ingeniería de la Universidad Autónoma de Querétaro (UAQ).Estudió la licenciatura de ingeniero electromecánico, la maestría en ciencias con especialidad en instrumentación y control y el doctorado en ingeniería en la UAQ. Las áreas de interés son visión artificial, procesamiento de señales y sistemas de supervisión aplicados a la manufactura.

Miguel Trejo-Hernández. Profesor de la Facultad de Ingeniería en la Universidad Autónoma de Querétaro (UAQ). Es ingeniero electromecánico, maestro en instrumentación y control automático y doctor en ingeniería por la UAQ. El control inteligente, las vibraciones mecánicas, el diseño y manufactura avanzada son algunas áreas de su interés. 